\title{
THE EFFECTIVENESS OF USING HUMAN PATIENT SIMULATION MANIKINS IN THE TEACHING OF CLINICAL REASONING SKILLS TO UNDERGRADUATE NURSING STUDENTS: A SYSTEMATIC REVIEW
}

\author{
Samuel Lapkin \\ BN University of Newcastle (2007), RN (NSW)
}

\author{
A thesis submitted in fulfilment of the requirements \\ for Bachelor of Nursing Honours Degree \\ in the School of Nursing and Midwifery, \\ Faculty of Health, \\ University of Newcastle, \\ New South Wales \\ September 2009
}


This thesis contains no material which has been accepted for the award of any other degree or diploma in any university or other tertiary institution and, to the best of my knowledge and belief, contains no material previously published or written by another person, except where due reference has been made in the text. I give consent to this copy of my thesis, when deposited in the University Library**, being made available for loan and photocopying subject to the provisions of the Copyright Act 1968.

**Unless an Embargo has been approved for a determined period.

(Signed)

Samuel Lapkin 


\section{Acknowledgments}

My sincere thanks go to my co-supervisors: Associate Professor Tracy Levett-Jones, who offered me a rare opportunity to be part of a major research project and Doctor Helen Bellchambers, whose ongoing support and encouragement was a source of strength. These two academics inspired me with their passion for research and continual search for knowledge. I literally experienced what it means to "stand on the shoulders of giants" (Bernard of Chartres, twelfth-century French Neo-Platonist philosopher, scholar, and administrator).

Special thanks to Doctor Ritin Fernandez, for agreeing to be the secondary reviewer and for offering critical feedback and support to this project. Most importantly I'd like to acknowledge the other members of the Australian Learning and Teaching Council project team: Dr Kerry Hoffman, Dr Sharon Bourgeois, Dr Sharyn Hunter, Dr Jennifer Dempsey, Dr Sarah Jeong, Noelene Hickey, Carol Norton, Raelene Kenny, and Karen Jeffrey.

Finally, to my special family members in Africa: my mum Winnet, brother Lucky, and his son LeeRoy and my sister Pamela, you give me the strength to go on. 


\section{Table of Contents}

Acknowledgments .......................................................................................................................3

List of Appendices...............................................................................................................6

1 Abstract.................................................................................................................

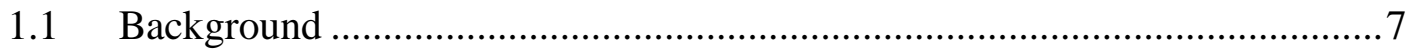

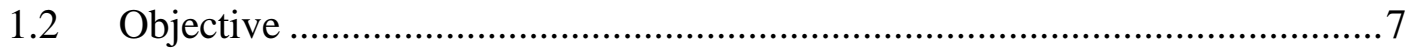

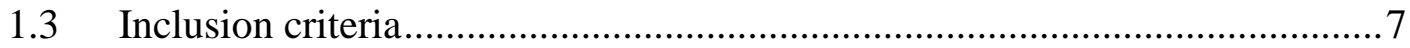

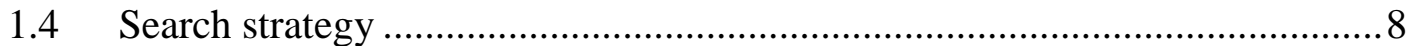

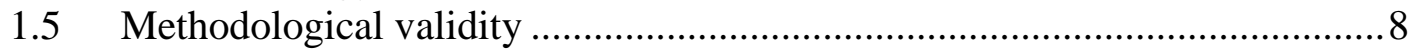

1.6 Data collection and synthesis ...................................................................

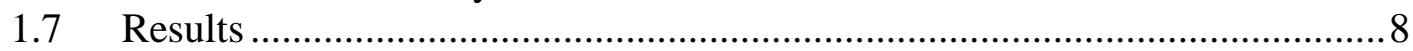

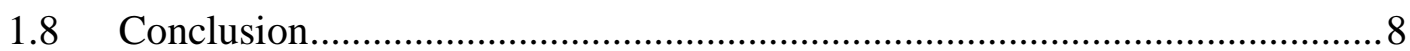

2 Definition of terms ......................................................................................................10

3 Glossary of Statistical Symbols and Terms ......................................................12

4 Introduction.............................................................................................................13

5 Background .......................................................................................................................14

6 Research question ...........................................................................................16

$7 \quad$ Research aim ................................................................................................................17

8 Ethical considerations..................................................................................................17

9 Review methods...................................................................................17

$9.1 \quad$ Inclusion criteria ....................................................................................

9.1.1 Type of studies ................................................................................. 17

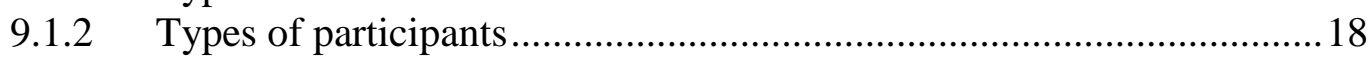

9.1.3 Types of intervention(s)/phenomena of interest ...................................18

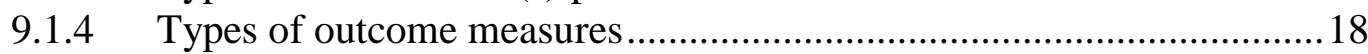

$10 \quad$ Search Strategy .............................................................................................................18

11 Assessment of methodological quality............................................................20

12 Data extraction ...........................................................................................................21

13 Data synthesis .............................................................................................................21

14 Data analysis............................................................................................21

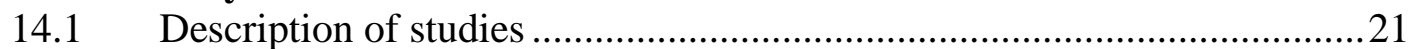

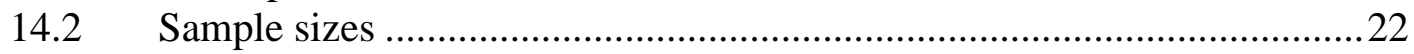

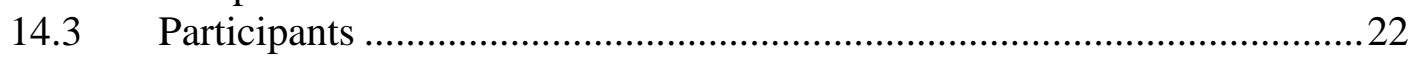

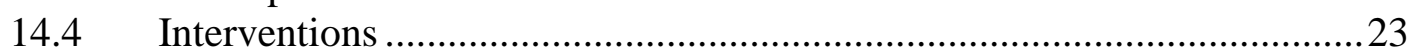

15 Methodological quality ……........................................................................................23

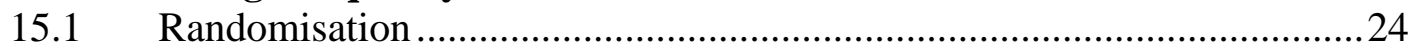

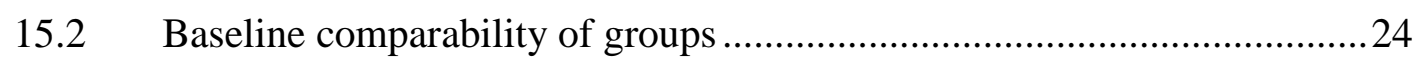

$15.3 \quad$ Blinded outcome assessment .................................................................24

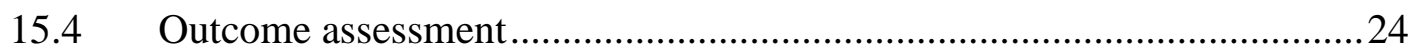

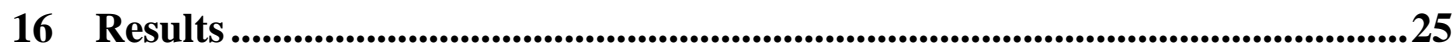

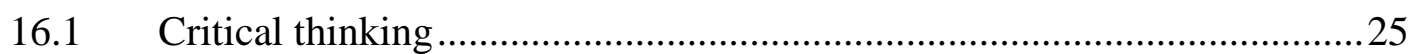

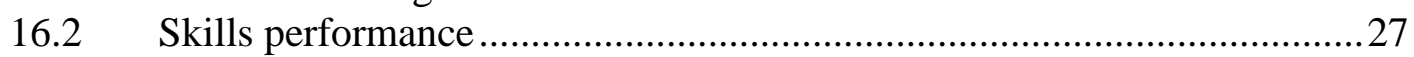

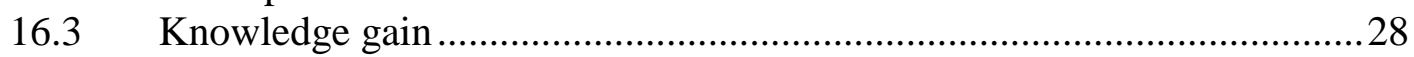

16.4 Self-reported levels of stress, confidence and judgment ...........................29

16.5 Student satisfaction with simulation experience ………………………........30

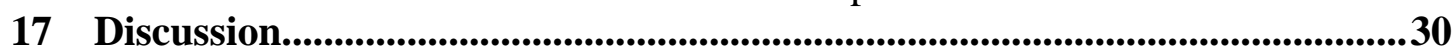

$17.1 \quad$ Clinical Reasoning .............................................................................. 


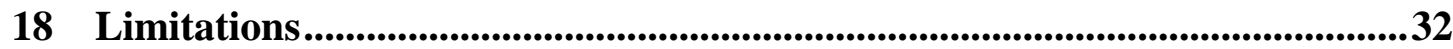

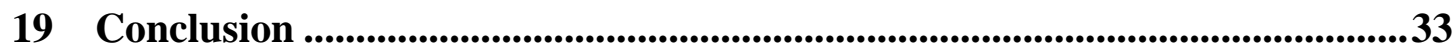

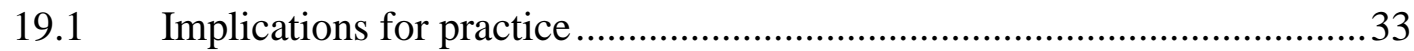

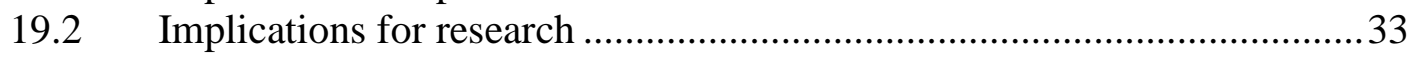

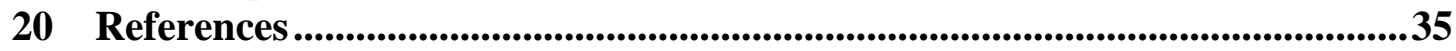




\section{List of Appendices}

Appendix I- Search Strategy ................................................................. 41

Appendix II: Summary of the search strategy...........................................p. 47

Appendix III - Critical Appraisal instruments for Experimental Studies......p. 48

Appendix IV - Data extraction instrument for Experimental Studies............p. 49

Appendix V-Excluded studies and reasons for exclusion............................p. 40

Appendix VI: Quality assessment of included studies................................ .p. 53

Appendix VII: Characteristics of included studies..........................p. 54

Appendix VIII: Considered Criteria for Honours Thesis Assessment............p. 59 


\section{Abstract}

\subsection{Background}

Nurses with effective clinical reasoning skills have a positive impact on patient outcomes. Conversely, those with poor clinical reasoning skills often fail to detect impending patient deterioration thus compromising patient safety. Human patient simulation manikins are being used extensively both nationally and internationally in the education of health professionals. There is evidence suggesting that these types of technologies are effective in teaching psychomotor skills and student satisfaction with simulation approaches is generally high. However, the extent to which human patient simulation manikins are effective in the teaching of clinical reasoning skills to undergraduate nursing students is less clear.

\subsection{Objective}

The aim of this systematic review was to identify the best available evidence on the effectiveness of using whole-body high-fidelity human patient simulation manikin to teach clinical reasoning skills to undergraduate nursing students.

\subsection{Inclusion criteria}

The review included all randomised controlled trials that assessed the effectiveness of high fidelity human patient manikins in educating undergraduate nursing students. Studies that included health professionals were excluded unless data for nursing students were analysed separately. The primary outcome measure was clinical reasoning, as assessed by methods such as objective structured clinical examinations and questionnaires. Other outcome measures included student satisfaction, knowledge acquisition, and psychomotor skill performance. 


\subsection{Search strategy}

Using a systematic search strategy designed for each database, the following electronic databases were searched for the period 1999 -2009: CINAHL, Cochrane Database, Dissertation and Theses, EMBASE, ERIC, MEDLINE, Ovid database, Proquest Nursing Journals, PsycINFO. Hand searching of the reference lists of included studies and conference proceedings were undertaken to identify further studies.

\subsection{Methodological validity}

Two independent reviewers' assessed the methodological quality of each study selected for retrieval prior to inclusion using the critical appraisal tool from the Joanna Briggs Institute.

\subsection{Data collection and synthesis}

Data were extracted from studies using the standardised data extraction tool from Joanna Briggs Institute. Due to the quality of available studies, statistical pooling was not possible and the findings are therefore presented in narrative form.

\section{$1.7 \quad$ Results}

Eight studies were selected for inclusion in this review. The results indicate that the use of human patient simulation manikins improves knowledge acquisition and enhanced students' satisfaction with the learning. There is lack of unequivocal evidence on the effectiveness of using high-fidelity human patient simulation manikins in the teaching of clinical reasoning skills to undergraduate nursing students.

\subsection{Conclusion}

Further research is required to ascertain the effectiveness of the use of human patient simulation manikins as an educational strategy to improve clinical reasoning skills of 
undergraduate nursing students. The importance of this research is underscored by the potential for patient outcomes to be improved through improved clinical reasoning skills in graduate nurses. 


\section{Definition of terms}

\section{Clinical reasoning}

In the nursing literature, terms such as clinical reasoning (CR), clinical judgement, problem solving, decision-making and critical thinking are frequently used interchangeably (Tanner, 2006; Thompson \& Dowding, 2002). For the purpose of this review, the term CR will be defined as the process by which nurses collect cues; process the information; come to an understanding of a patient problem or situation; plan and implement interventions; evaluate outcomes and reflect on and learn from the process (Hoffman, 2007; Levett-Jones, et al., in press; Tanner, Padrick, Westfall, \& Putzier, 1987).

\section{Fidelity}

Fidelity refers to the extent to which the simulation model resembles a live human.

\section{Low fidelity human patient simulation manikins}

Low fidelity HPSMs are static models or task trainers primarily comprised of rubber body parts which are used to practice of clinical skills such as intravenous cannulation, urinary catheterisation and basic life support (Issenberg, Gordon, Gordon, Safford, \& Hart, 2001; Seropian, Brown, Gavilanes, \& Driggers, 2004).

\section{Medium fidelity human patient simulation manikins}

Medium fidelity human patient simulation manikins (HPSMs) are full body manikins that have embedded software and can be controlled by an external, hand held device. They have more realism than the low-fidelity HPSMs. An example is Laerdal's Nursing Anne ${ }^{\mathrm{TM}}$ with VitalSim capability, a manikin used in nursing education to introduce and develop more complex skills such as auscultation of heart, breath and 
bowel sounds and identification of life-threatening cardiac dysrhythmias using electrocardiograph (Seropian, et al., 2004).

\section{High fidelity human patient simulation manikins (HPSMs)}

High fidelity HPSMs are life sized computerised manikins with realistic anatomical structures and high response fidelity (Alinier, Hunt, Gordon, \& Harwood, 2006). They can mimic diverse parameters of human anatomical physiology, for example changes in cardiovascular, pulmonary, metabolic and neurological systems, and have the ability to respond to nursing or pharmacological interventions in real time (Beyea \& Kobokovich, 2004; Holcomb, et al., 2002; Nehring, Lashley, \& Ellis, 2002; Seropian, et al., 2004). Examples of HPSMs include Laerdal SimMan Universal Patient Simulator $\left(\operatorname{SimMan}^{T M}\right)$ and $\operatorname{METI}^{T M}$ manikins.

\section{Simulation}

Although there are numerous definitions of simulation, the one described by Gaba has been adopted for this review. Gaba (2007) defines simulation as a technique used "to replace or amplify real experiences with guided experiences that evoke or replicate substantial aspects of the real world in a fully interactive manner" (p. 126). 


\section{Glossary of statistical symbols and terms}

\begin{tabular}{lll} 
Symbol & Term & Description \\
\hline$p$ & probability value & The probability that a statistical result would occur \\
& by chance if a NULL hypothesis was true. When \\
& probability values are less than .05, observed scores \\
& can be described as "significantly different" since \\
& there is a low likelihood of obtaining these \\
& observed scores by chance alone. \\
N & sample size & Total number in sample \\
SD & Atandard deviation & A measure of the spread/dispersion of scores \\
& & around the mean score. \\
&
\end{tabular}

